\title{
Providing the Best Care for Patients with Breast Cancer Through Use of the Multidisciplinary Team
}

\author{
Judy C. Boughey, $\mathrm{MD}^{1}$ and Jill Dietz ${ }^{2}$ \\ ${ }^{1}$ Department of Surgery, Mayo Clinic, Rochester, MN; ${ }^{2}$ University Hospitals Seidman Cancer Center, Case Western \\ Reserve University, Cleveland, $\mathrm{OH}$
}

The 15th Annual Meeting of the American Society of Breast Surgeons (ASBrS) held in May in Las Vegas was host to an audience of 1,460 , including 1,370 physicians (187 from 35 countries outside of the US) as well as an esteemed panel of experts in breast surgery and across the multidisciplinary field of breast diseases. There was extensive discussion on surgical issues, such as the utility of axillary dissection, management of ductal carcinoma in situ, new surgical techniques, and appropriate margin status. The expertise imparted by many of our non-surgical colleagues highlighted the importance of a strong multidisciplinary team in order to provide the best care to our cancer patients. Multidisciplinary care is a critical part of the clinical practice for breast patients. Classically, when thinking about multidisciplinary teams for breast cancer the make-up can vary but commonly includes input from breast surgery, medical oncology, radiation oncology, pathology, and breast imaging, as represented in the always popular 'community tumor board'. However, expertise from other fields can also augment and improve care of breast patients. The recent meeting included input from anesthesiologists, integrative medicine physicians and reconstructive surgeons.

Breast radiologists are an integral part of screening and diagnostic work-up of breast patients. The debate surrounding mammograms for screening continues and was eloquently reviewed by a panel of experts. In 2009, the US Preventative Services Task Force (USHPTF) recommended that screening for breast cancer in the US should

\section{(C) Society of Surgical Oncology 2014}

First Received: 15 July 2014;

Published Online: 6 August 2014

J. C. Boughey, MD

e-mail: boughey.judy@mayo.edu start at 50 years of age and continue every other year thereafter. ${ }^{1}$ Many breast surgeons agree that starting annual screening in every patient at 40 years of age is unnecessary, expensive, and associated with some risk; however, most medical organizations, including the ASBrS, continue to recommend annual mammography for all women starting at 40 years of age. This is because there is no current, compelling risk model that can accurately stratify young women into a high or low risk group. Moving forward, it may well be that a one size fits all approach is not ideal and consideration is underway for a risk-stratified approach to screening, so women at higher risk of breast cancer development start screening earlier and potentially more frequently than women at average or below-average risk.

Breast surgeons work closely side by side with plastic and reconstructive surgeons. Some oncoplastic procedures can be performed by breast surgeons, such as local tissue rearrangements, the round block resection (donut mastopexy) lumpectomy, and parallelogram mastopexy lumpectomy. Experience with and indications for nipple-sparing mastectomy has grown over the last 5-10 years and the group from the University of California San Franciso (USCF) presented the evolution of their techniques over 986 cases, with the article included in this issue of Annals of Surgical Oncology. Immediate or delayed lipofilling is a newer technique used to improve both postmastectomy and postlumpectomy deformities. This simple technique performed without creating a visible scar brings fat removed by liposuction from the subcutaneous tissue from the abdomen or other donor sites on the patient's body to fill the defects after surgery. The degree of reabsorption of the injected adipose tissue is unpredictable. Long-term oncologic follow-up related to lipofilling is not available; however, early studies indicate that lipofilling seems to be a safe procedure for breast cancer patients. ${ }^{2,3}$ Plastic and reconstructive surgeons offer a whole variety of procedures from immediate and delayed 
reconstruction of mastectomy defects to reshaping and repair of lumpectomy defects, and also, in some cases, lymphatic transfer for the treatment of lymphedema. Having a team and knowledge of the options available for patients can ensure that patients are aware of all available options as they make their decisions for both their cancer care and their long-term cosmesis.

Anesthesia is not a discipline commonly considered as part of the multidisciplinary team for breast patients; however, with enhanced recovery pathways and regional anesthesia techniques, the anesthesiologists significantly impact the patient's perioperative experience. Regional anesthesia techniques such as paravertebral block can decrease postoperative nausea and vomiting, ${ }^{4}$ and potentially also decrease hospital length of stay ${ }^{5}$ and chronic pain. The addition of clonidine to paravertebral blocks enhances the analgesic efficacy up to 3 days postoperatively for patients having breast surgery. ${ }^{6}$ Some literature has also suggested improved oncologic outcomes from the use of paravertebral blocks in breast cancer surgery, although further evaluation of this possibility is needed. Intraoperative use of non-steroidal anti-inflammatory drugs (NSAIDs) has also been indicated to impact disease-free survival and overall survival after conservative breast cancer surgery. ${ }^{7}$ Perioperative pain management techniques influence the body's stress response and immune suppression response, and this can potentially impact tumor suppression. Although some small studies to date have indicated that intraoperative analgesics may influence breast cancer recurrence, there is currently insufficient evidence to recommend any anesthesia technique specifically to decrease cancer recurrence risk.

The systemic therapy landscape for breast cancer patients has evolved significantly over the last 5-10 years. Where previously breast surgeons were comfortable telling patients with breast cancer that indications for chemotherapy were based on tumor size and nodal involvement and drugs utilized were anthracyclines with cyclophosphamide followed by taxanes, it no longer remains so simple or predictable. The decision for chemotherapy is based on tumor biology more than size and lymph node involvement. Drugs chosen vary by tumor type, with the consideration of platinum therapy for triple-negative disease, use of trastuzumab for HER2-neu positive disease and now, most recently, consideration of Perjeta in the neoadjuvant setting for HER2-neu positive disease. The neoadjuvant space provides opportunities to advance the field of breast oncology using pathologic complete response rates at surgery as a surrogate for survival and enabling studies to require significantly fewer patients a shorter time to obtain results. This therefore provides us with a pathway to accelerated approval of novel drugs and drug combinations. Studying the tumor at timepoints through neoadjuvant therapy can help identify mechanisms of drug resistance, and molecular profiling can help identify pathways for novel drug development. Understanding which patients benefit from neoadjuvant chemotherapy also helps us identify those where chemotherapy may not be as beneficial. In many of these patients, especially for luminal tumors, neoadjuvant endocrine therapy is being increasingly utilized. The breast surgeon has a pivotal role in understanding and presenting the concept of neoadjuvant therapy to patients and potentially offering participation in a neoadjuvant clinical trial. Tissue samples are key for all studies to further understanding of the response to therapy and for molecular profiling, and surgeons have access to the tissue.

Radiation oncologists provided insight into another controversial topic regarding the survival benefits of radiation therapy in early-stage breast cancer. Adjuvant radiotherapy has been shown to improve overall survival for women with invasive breast cancer, for patients undergoing breast-conserving surgery and for those with high-risk node positive disease undergoing mastectomy. The lower the likelihood of recurrence, the less the benefit from adjuvant radiation. Therefore, the better that surgical resection and systemic therapy gets, re-evaluation of which patients benefit from radiation is valuable. Local relapse rates of $10-20 \%$ are not commonly seen in the current era, therefore the reduction in relapse rates will not be as dramatic. In addition, the role of partial breast irradiation and intraoperative radiation are expanding and these techniques are being increasingly utilized. This provides for us further demonstration that evaluating the impact of all disciplines on the patient's cancer therapy remains crucial. When deciding for or against radiation therapy in addition to the surgical procedure performed, we should also consider whether patients will take adjuvant endocrine therapy and thus their risk of recurrence with and without radiation.

Margin recommendations and margin assessment has been a hot topic with the recent publication of Surgical Society of Oncology and American Society for Radiation Oncology consensus guidelines on margins for breast cancer. ${ }^{8}$ These guidelines recommend no ink on tumor as the standard for an adequate margin in invasive cancer. An exciting session reviewed the guidelines and presented the pathologist perspective as well as the methods of intraoperative margin assessment used by surgeons and impacts on margins. We recommend the article on this session included in this issue of Annals of Surgical Oncology. ${ }^{9}$ Review of data from the ASBrS Mastery of Breast Surgery data presented highlighted the reasons for re-excision after lumpectomy for breast cancer and provides insight in treatment patterns. Of all re-excisions, $49.7 \%$ were for margins positive by tumor on ink, with $34.3 \%$ due to margins $<1 \mathrm{~mm}$ and $7.2 \%$ for margins of $1-2 \mathrm{~mm}$. The re- 
excision lumpectomy rate was lower in surgeons with high procedure volume. This presentation by Dr. Jeffrey Landercasper received the Scientific Impact Award, with the article included in this issue Annals of Surgical Oncology. ${ }^{10}$

Other awards presented at the meeting with articles included in this issue of Annals of Surgical Oncology were (1) the George Peters Award for the best abstract presented by a breast fellow, which was awarded to Erin Cordeiro, MD, for her presentation of "Is Breast Surgery for Metastatic Breast Cancer Safe? An Analysis of the NSQIP Database"; ${ }^{11}$ and (2) the Scientific Recognition Award to Andrea Covelli, MD, $\mathrm{PhD}$, for her presentation on "Increasing Mastectomy Rates: The Role of the HealthCare Milieu. A Comparative Analysis Between Canada and the United States". ${ }^{2}$

Providing care for patients with breast disease can be complex and require a multifaceted approach. Working with and understanding what all the disciplines of the multidisciplinary team brings to the table allows the surgeon to refer appropriate patients to appropriate disciplines and set up patients' expectations appropriately for a smooth and comprehensive experience for the patient. Working together, the team is greater than the sum of its parts.

We hope you enjoy this issue of Annals of Surgical Oncology, providing in-print articles from some of the key sessions as well as articles from some of the oral sessions, quickshot sessions, and poster sessions from the ASBrS meeting earlier this year, and we look forward to seeing you at the annual meeting in Orlando in April-May 2015.

\section{REFERENCES}

1. US Preventive Services Task Force. Screening for breast cancer: US Preventive Services Task Force recommendation statement. Ann Int Med. 2009;151(10):716-726, W-236.

2. Petit JY, Lohsiriwat V, Clough KB, et al. The oncologic outcome and immediate surgical complications of lipofilling in breast cancer patients: a multicenter study-Milan-Paris-Lyon experience of
646 lipofilling procedures. Plast Reconstr Surg. 2011;128(2): 341-346.

3. Petit JY, Botteri E, Lohsiriwat V, et al. Locoregional recurrence risk after lipofilling in breast cancer patients. Ann Oncol. 2012;23(3):582-588.

4. Fahy AS, Jakub JW, Benzon MD, et al. Paravertebral blocks in patients undergoing mastectomy with or without immediate reconstruction provides improved pain control and decreased postoperative nausea and vomiting. Ann Surg Oncol. 2014. doi:10.1245/s10434-014-3923-z.

5. Coopey SB, Specht MC, Warren L, Smith BL, Winograd JM, Fleischmann K. Use of preoperative paravertebral block decreases length of stay in patients undergoing mastectomy plus immediate reconstruction. Ann Surg Oncol. 2013;20(4):12821286.

6. Naja ZM, Ziade FM, El-Rajab MA, Naccash N, Ayoubi JM. Guided paravertebral blocks with versus without clonidine for women undergoing breast surgery: a prospective double-blinded randomized study. Anesth Analg. 2013;117(1):252-258.

7. Forget P, Bentin C, Machiels JP, Berliere M, Coulie PG, De Kock $M$. Intraoperative use of ketorolac or diclofenac is associated with improved disease-free survival and overall survival in conservative breast cancer surgery. Br J Anaesth. 2014;113(suppl 1):i82i87.

8. Moran MS, Schnitt SJ, Giuliano AE, et al. Society of Surgical Oncology-American Society for Radiation Oncology consensus guideline on margins for breast-conserving surgery with wholebreast irradiation in stages I and II invasive breast cancer. Ann Surg Oncol. 2014;21(3):704-716.

9. Harness JK, Giuliano AE, Pockaj BA, Downs-Kelly E. Margins-a status report from the Annual Meeting of the American Society of Breast Surgeons. Ann Surg Oncol. 2014. doi:10.1245/ s10434-014-3957-2.

10. Landercasper J, Whitacre EB, Degnim AC, Al-Hamadani M. Reasons for re-excision after lumpectomy for breast cancer: insight from the American Society of Breast Surgeons Mastery ${ }^{\mathrm{SM}}$ Database. Ann Surg Oncol. 2014. doi:10.1245/s10434-0143905-1.

11. Cordeiro E, Jackson TD, Elanhas A, Cil T. Higher rate of breast surgery complications in patients with metastatic breast cancer: an analysis of the NSQIP database. Ann Surg Oncol. 2014. doi:10.1245/s10434-014-3959-0.

12. Covelli AM, Baxter NN, Fitch MI, Wright FC. Increasing mastectomy rates-the role of the health-care milieu: a comparative analysis between Canada and the United States. Ann Surg Oncol. 2014. doi:10.1245/s10434-014-3955-4. 